\title{
Improving Retinal Image Quality Using the Contrast Stretching, Histogram Equalization, and CLAHE Methods with Median Filters
}

\author{
Erwin \\ Computer Engineering, Sriwijaya University, Indralaya, Indonesia \\ Email: erwin@unsri.ac.id \\ Dwi Ratna Ningsih \\ Computer Engineering, Sriwijaya University, Indralaya, Indonesia \\ Email: dwirn20@gmail.com
}

Received: 01 December 2019; Accepted: 15 March 2020; Published: 08 April 2020

\begin{abstract}
This paper performs three different contrast testing methods, namely contrast stretching, histogram equalization, and CLAHE using a median filter. Poor quality images will be corrected and performed with a median filter removal filter. STARE dataset images that use images with different contrast values for each image. For this reason, evaluating the results of the three parameters tested are; MSE, PSNR, and SSIM. With the gray level scale image and contrast stretching which stretches the pixel value by stretching the stretchlim technique with the MSE result are 9.15, PSNR is 42.14 $\mathrm{dB}$, and SSIM is 0.88. And the HE method and median filter with the results of the average value of MSE is 18.67, PSNR is $41.33 \mathrm{~dB}$, and SSIM is 0.77 . Whereas for CLAHE and median filters the average yield of MSE is 28.42, PSNR is $35.30 \mathrm{~dB}$, and SSIM is 0.86. From the test results, it can be seen that the proposed method has MSE and PSNR values as well as SSIM values.
\end{abstract}

Index Terms-Median filter, STARE, contrast stretching, CLAHE, HE, PSNR, SSIM.

\section{INTRODUCTION}

Enhancement of the quality of retinal images that have both noise and noise is the first step in image processing to help improve the accuracy of the results for image segmentation and extraction. Images store a lot of information, but often there is a decrease in quality or image defects. So that images that have experienced interference or noise are easily interpreted, then the image can be manipulated into other images of better quality using image processing techniques or methods[1]. Retinal eye images can provide information about pathological changes caused due to disease[2]. Processing medical image of the retina in the eye with disease information by increasing contrast quality and eliminating noise that can damage the image so that sharpness can be improved and can facilitate segmentation or extraction of retinal images
[3]. From an image, sharp lines can be sharpened to increase. Poor and poor image quality cannot be interpreted directly by the human eye, so we use methods to improve the quality of contrast stretching images and median filters[4]. One type of image modeling used for digital processing, one of which has good results is RGB. To find out the color frequency in a digital image a histogram graph is used[5]. In the image, there are bright and dark noise in the random distribution in the image which can affect the visual effect of the image as well as the information in the image[6]. Then the method to improve the image quality of the median filter and contrast stretching is applied. It is a method that will increase contrast by getting new images as well as new contrast.

In this pre-processing stage, two contrast methods are used namely contrast stretching and histogram equalization and CLAHE. Increase contrast by stretching the existing value in the image at a certain intensity. Contrast stretching will stretch, increase and decrease the contrast of the image. And the median filter as a filter for image enhancement that will filter out noise or noise. With this increase in image quality, it can be seen which method is better in improving it. With the parameters of PSNR, SSIM and MSE to see the image quality and whether or not accurate.

The test of this research is that it can improve the low image quality by processing the image with contrast stretching with the median filter method for noise removal. Obtaining better image results than the original image that has not been improved image quality. Limitation of the problem is limited by researchers, namely only discussing on improving image quality with the Contrast Stretching method using Stretchlim, HE and CLAHE. And noise removal with a median filter. By using the STARE dataset which is secondary data obtained through the STARE dataset website. Using the .jpg format. In this study, the authors only used 20 STARE datasets. Showing the results of image quality improvement using the parameters of Mean Square Error 
(MSE), PSNR (Peak Signal to Noise Ratio) and SSIM (Structural Similarity Index).

\section{RELATED WORK}

In previous studies[7] the original retinal image will be processed with a normalized convolution algorithm using domain transformation and obtain an enhanced image. With the median filter method using the STARE database to evaluate the results of image enhancement. The result of this method is that it can improve the quality of the retinal image is still unclear.

From previous studies[8] using edge detection and contrast enhancement to eliminate noise. This research focuses on stretching contrast and image enhancement. Use a histogram to verify the results of image enhancement and contrast stretching. Which increased quality is not optimal.

Previous studies[9] improved the perception of information that exists in the original image, increasing structural features for good image quality. With morphological and merging regions which will then be continued by stretching the contrast separately. Linear stretching is used to improve the texture features of objects, can suppress noise but still cannot maintain the brightness of the entire image.

Previous studies[10] increased contrast with morphological methods of upper and lower operations. Matrix contrast at edges extracts the enhanced image. Counting each region with morphological open operations, everything connected with pixels under 40 will be eliminated. But the difficulty in removing noise.

Previous studies[11] used methods to improve blood vessels. A multi-scale top-hat transformation extracts the original image into bright and dim image features. Then it is enhanced by adding an image feature that has a gap and a gaussian curve histogram is mounted for linear stretching.

Based on research conducted several factors can affect the results of the processing of an image at the time of quality improvement. Different contrast qualities make the test results in different based on the quality of each image. The filter used can affect the results of the image to be smoother than the initial image.

\section{METHOD AND RESULT}

This paper uses a database of stare in which the initial stage is to input a retinal image and then the image is given three RGB colors with an intensity of 8-bit depth. Separate each RGB color into three channels. RGB image or color image which consists of three color elements namely red (R), green $(\mathrm{G})$ and blue (B). Color component of RGB images have a resolution of 24 bits which uses eight bits (from 0 to 255) in each component, allows color mapping in space three dimension.

From RGB, the image is grayed with pixel intensity. On grayscale with a gray image that will be filtered with the median filter, then with contrast stretching, can be seen in the illustration in figure .1 .

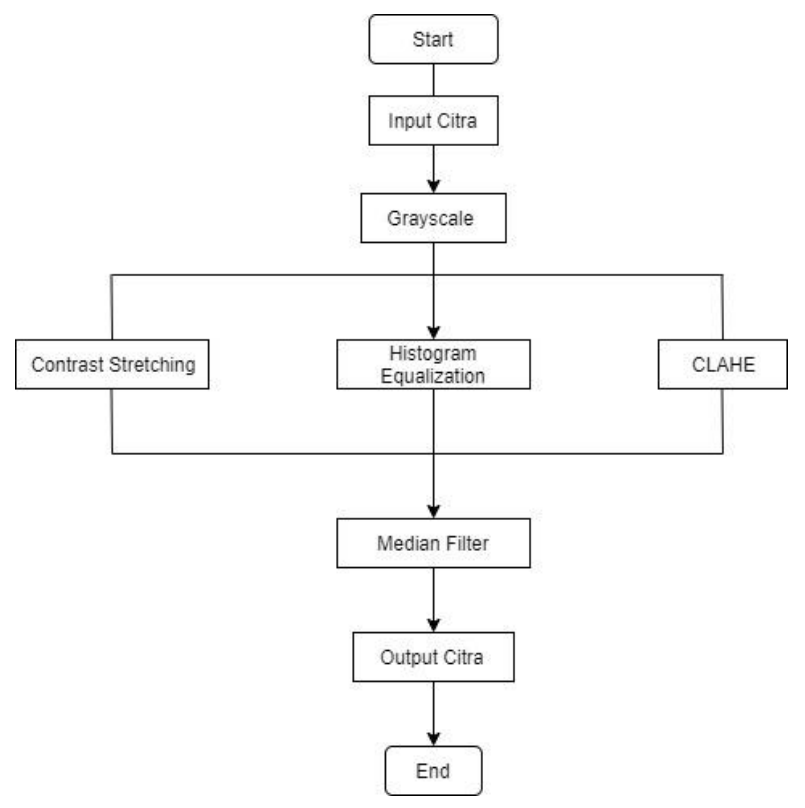

Fig.1. Method Diagram Block

At this stage of the process the first is to input the retinal image using the STARE database, after the median filter which will then be contrasted with contrast stretching which are the methods in step figure.1, as follows:

\section{A. Grayscale}

Gray pixel intensity, with values ranging from 0 to 255 expresses the gray range of the image. With this range between the gradations of black and white images. Grayscale is a color that is located between black and white. On this grayscale, the image is first increased in complexity. The original image before the grayscale image is an RGB image with three elements of red (R), green (G), and blue (B). With the formula[12]:

$$
\begin{aligned}
& \text { Grayscale }=(0,299 * \mathrm{R})+ \\
& (0,587 * \mathrm{G})+(0,114 * \mathrm{~B})
\end{aligned}
$$

Where,

$$
\begin{aligned}
& R=\text { red channel matrix value } \\
& G=\text { green channel matrix value } \\
& B=\text { blue channel matrix value }
\end{aligned}
$$

\section{B. Median Filter}

It is a non-linear method to filter or reduce noise in the image so that image information is maintained. Values on images will be replaced or manipulated by making values as medians. With the gray level, the value has been replaced by the median then stored instead of the noise value. The max is the maximum, the min is the minimum value, and the average is the gray level. And $y(m, n)$ is the center of manipulation of the median filter[13]. 


$$
y[m, n]=\operatorname{median}\{x[i, j],(i, j) \in \omega
$$

Where,

$\mathrm{y}[\mathrm{m}, \mathrm{n}]=$ matrix of results labeled $\mathrm{y}$ with $\mathrm{m}, \mathrm{n}$ is a row multiplied by a column.

$\{x[i, j],(i, j) \in \omega=$ matrix value of the image being processed or the corresponding elements sorted.

The median filter will see the pixel value of its neighbor and will match the pixel value that will be represented by the neighbor's pixel value. The pixel value will be replaced at the middle value with a count of around neighbors. When the value is even on the surrounding pixel, the middle value will be used with an average of two middle values[14].

\section{Histogram Equalization (HE)}

It is a histogram equalization in which the gray distribution of values is made flat. HE will manipulate the respective image pixels for increased contrast. The image gets a flattened histogram distribution function. The intensity and scale are obtained as output from the histogram equalization. The purpose of this $\mathrm{HE}$ is to make the distribution of the histogram evenly distributed so that the pixel value at the gray level will have a relatively identical value or the same. HE will map input images with unequal intensity levels and to output images with the same level[15].

\section{Contrast Stretching (CS)}

It is a method of stretching the contrast, with the intensity value contained in the image expanded with a dynamic range. The pixel value of the image can be applied with linear scaling. To normalize the image or contrast stretching the image it is necessary to determine the minimum and maximum values of the image. These minimum and maximum values will determine the boundary of the image. In this proposed method an image with an 8-bit gray level is the lower limit and values 0 to 255 as the upper limit. Digital images are taken using a fundus camera. When viewed from the outline angle, digital image processing techniques are divided into 3 based on processing levels, namely:

1. Low-Level Process or low level, this processing is a basic operation in image processing examples such as noise reduction, image improvement, and image restoration.

2. Mid-Level Process or intermediate level, this processing includes object description, image segmentation, and object classification separately.

3. High-Level Process or high level includes the analysis of an image.[16].

$$
g(\mathrm{x}, \mathrm{y})=\frac{f(x, y)-\min }{\max -\min } X 255
$$

Where,

$\mathrm{g}(\mathrm{x}, \mathrm{y})=$ matrix of the resulting image

\section{$\mathrm{f}(\mathrm{x}, \mathrm{y})=$ original image matrix value}

Where $g(x, y)$ represents the output and $f(x, y)$ represents the input. Image intensity values with 0 as the lowest value and 255 as the highest value[17]. By using stretchlim as a determinant of the minimum and maximum values. The value of $\mathrm{g}(\mathrm{x}, \mathrm{y})$ as a new image obtained from the image value $(\mathrm{x}, \mathrm{y})$ will be subtracted by the maximum value and divided by the results of the minimum and maximum reduction. The results will be multiplied by 255 as the pixel value[18]

\section{E. CLAHE(Contrast Limited Adaptive Histogram Equalization)}

CLAHE is a contrast enhancement technique which is the development of $\mathrm{HE}$ and AHE from which surely this CLAHE is better than HE and AHE. In this CLAHE images will be divided into tiles or smaller areas. CLAHE is also a development of the AHE method that has its components changed. In this CLAHE all pixels adjacent to all functions will be converted[14]. This CLAHE is different from AHE because CLAHE limits the contrast, uses the maximum value on the clip and returns it to the gray value [19].

\section{F. Mean Square Error (MSE) and PSNR (Peak Signal to Noise Ratio)}

The parameters used as an evaluation of the results of improving image quality. This parameter will compare the original image with the resulting image, the equation used is as follows[20] :

$$
M S E=\frac{1}{X Y} \sum_{y=0}^{Y=1} \sum_{x=0}^{X=1}(\hat{y}(i, j)-y(i, j))^{2}
$$

Where,

$$
y^{\wedge}(i, j)=\text { matrix of the yield image }
$$$$
y(i, j)=\text { original image matrix value }
$$

$$
P S N R=10 \log _{10} \frac{s^{2}}{M S E}
$$

With the value $S=255$ for 8 -bit images, the higher the PSNR value generated, the better. While the closer the value to 0 , the better the MSE value.

\section{G. SSIM (Structural Similarity Index)}

SSIM is a parameter used for the evaluation of results using quantitative measures. The value of SSIM is obtained by dividing the original image with a distorted image which is then converted into a vector. With the following formula equation[21]:

$$
S S I M=\frac{\left(2 \mu_{x} \mu_{y}+c_{1}\right)\left(2 \sigma_{x y}+c_{2}\right)}{\left(\mu_{x}^{2}+\mu_{y}^{2}+c_{1}\right)\left(\sigma_{x}^{2}+\sigma_{y}^{2}+c_{2}\right)}
$$

Where,

$\mu \_\mathrm{x}=$ average value of $\mathrm{X}$

$\mu \_y=$ average value of $Y$ 
$\sigma \_x y=$ correlation coefficient on $\mathrm{X}$ and $\mathrm{Y}$

$\sigma \_\mathrm{X}^{\wedge} 2=$ difference in values on $\mathrm{X}$

$\sigma \_\mathrm{y}^{\wedge} 2=$ difference in values on $\mathrm{Y}$

c_1 and c_2 = two variables that stabilize the low denominator

The more SSIM values close to zero, the better the improved image.

\section{H. Result Enhancement}

MATLAB R2018b was used in the course of this research, which is managed by a laptop device with an AMD Quad-Core A10-9620P processor, up to $3.4 \mathrm{GHz}$ with $4 \mathrm{~GB}$ of RAM. By using the STARE database taken, there were 20 images. Which uses JPG format. The image used is a dataset from STARE (Structured Analysis of the Retina).

Inputting the image with the original image in the first stage can be seen in figure 1 . The median filter will manipulate the image to get better image quality improvements. Then the shortcomings of the median filter will be followed by contrast stretching which will increase the contrast with level to gray.

By using the equation formula (1), the grayscale results from the original RGB image by taking the pixel value can be seen as follows:

$$
\begin{aligned}
& G=163 \\
& B=165 \\
& R=193
\end{aligned}
$$

Grayscale $=(0,299 * \mathrm{R})+(0,587 * \mathrm{G})+(0,114 * \mathrm{~B})$ $=(0.299 * 193)+(0.587 * 163)+(0.114 * 165)$ $=172,198$

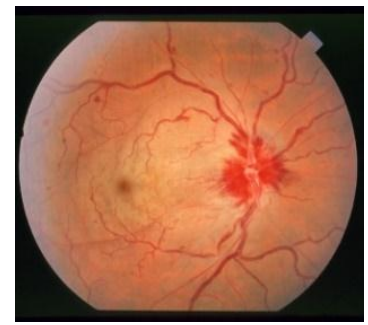

(a)

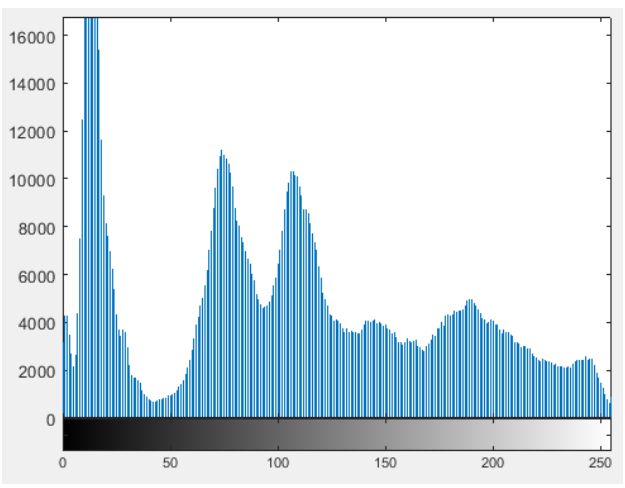

(c)

Fig.2. a. Original image b. Grayscale c. Original image histogram

Figure 2 uses im0005.jpg which is in part (a) the original image from the STARE database. Based on Figure 2 (b) is the original input image of the dataset that is made grayscale or to a grayscale. Figure 2 (c) is a histogram of the original image that has not been processed. By determining the lower limit and the pixel value, the image is normalized. Suppose that a is the lower limit and $\mathrm{b}$ is the upper limit, then find the highest and lowest pixels of the image. With $\mathrm{c}$ and $\mathrm{d}$, take the lowest and highest pixel values, and scale the pixels by the following equation:

$$
P \text { out }=(P \text { in }-c) \frac{(b-a)}{(d-c)}+a
$$

Values $c$ and d can affect the output value especially if the value is too high or low.

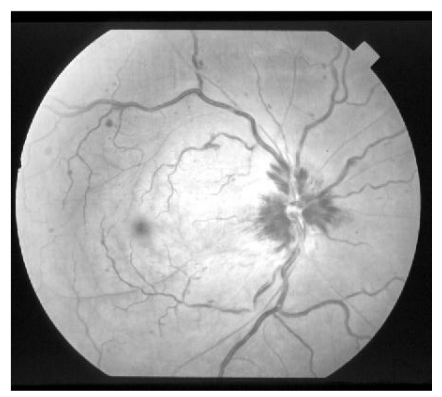

Fig.3. Contrast Stretching Using Stretchlim image STARE im0005.jpg

Figure 3 is the result of the graying image normalized by contrast stretching and stretching methods. Stretching will normalize the image by looking for the minimum and maximum values first. Stretchlim will look for maximum and minimum values of gray-level images.

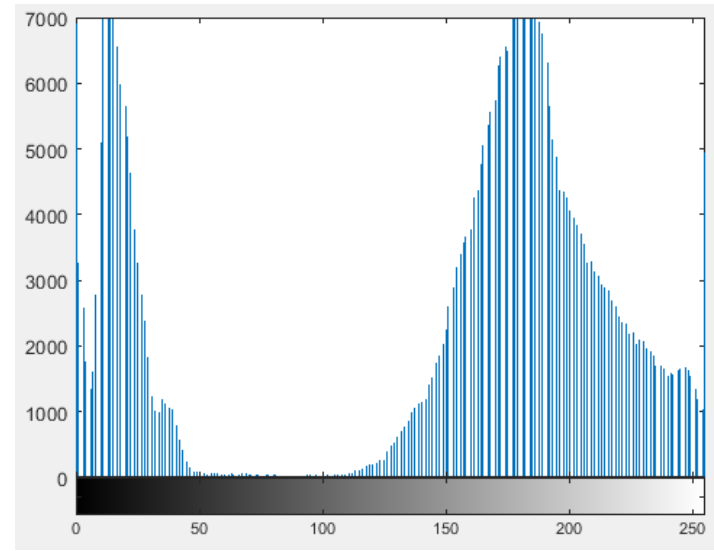

Fig.4. Histogram Contrast Stretching Using Stretchlim image STARE im0005.jpg

Figure 4 displays the histogram of contrast stretching with the results seen from the histogram that the image is stretching. By stretching the intensity value of the image can be seen if the counter is too far to the right with a pixel value above 135 then the image is included as bright. Conversely, if the cons on the histogram are more left or under 50 pixels based on the histogram, the image is dark. In the histogram, the image is more dominant towards the light.

Figure 5 is the image of im005.jpg which has done even distribution of the histogram with HE. The image in a gray level that is made flat or identical. In the image 
visible difference from the results of contrast stretching images and median filters that blood vessels are more visible in the HE method and median filter.

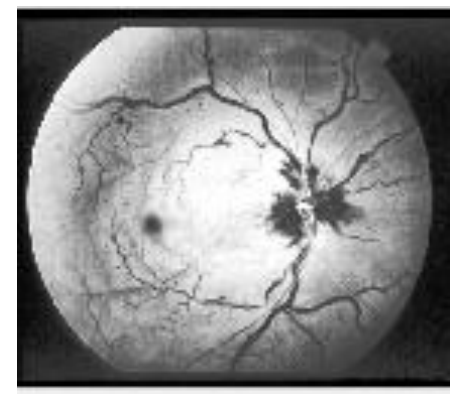

Fig .5. HE image im0005.jpg

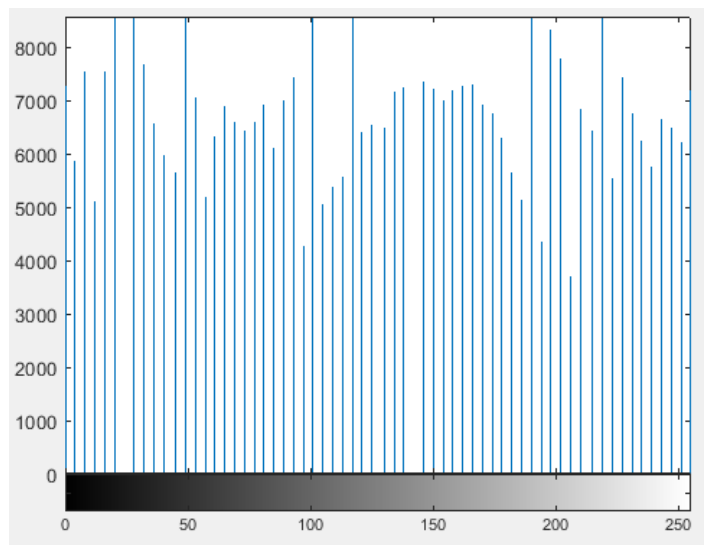

Fig.6. Histogram with HE image im0005.jpg

Figure 6 is a histogram of the im005.jpg image leveled by the HE method. From the original image histogram, the difference between the histogram results of HE which is more even and equal. With a range of 0 to 255 and the grayscale histogram is made uniform and obtained evenly distributed.

The results of CLAHE and median filters can be seen in Figure 7 where all pixels are converted, and the contrast value is limited to the maximum value. Seen in the image that this CLAHE method can highlight the small blood vessels of the retina of the eye compared to contrast stretching and HE.

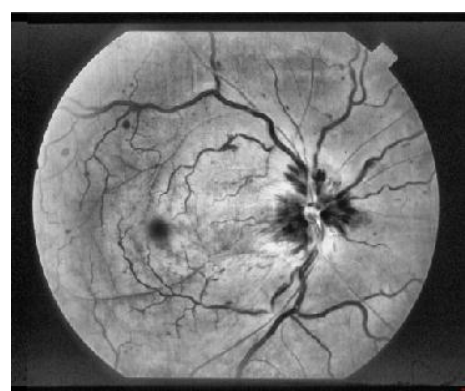

Fig.7. CLAHE image im0005.jpg

This paper uses three parameters, namely MSE and PSNR to assess the results of the method being tested. The testing method was carried out on a 4GB ASUS X555Q laptop. The results of MSE can be seen in table 2 . And the results of PSNR can be seen in table 3. And the results of SSIM can be seen in table 4 .

The results table 1 is obtained by testing on 20 STARE dataset images with a contrast enhancement method and median filter that will refine the image. The accuracy of the test results can be seen from the measurement parameters used, namely MSE, PSNR, and SSIM. Where the MSE value is getting closer to the value of 0 , the better it means that the smaller the error value. While PSNR will state the value of image processing results if the image is more accurate if the PSNR value exceeds 35 $\mathrm{dB}$ and vice versa if the PSNR value is below $35 \mathrm{~dB}$ then the value of the image processing result is not too good. While the SSIM value will be said to be accurate if the value of the image processing results close to 1 is said to be more accurate. SSIM value is in the range of $0-1$ if the SSIM value is close to 0 then it can be said that the image processing results are not good enough.

Table 1. The results of 20 retinal datasets were tested

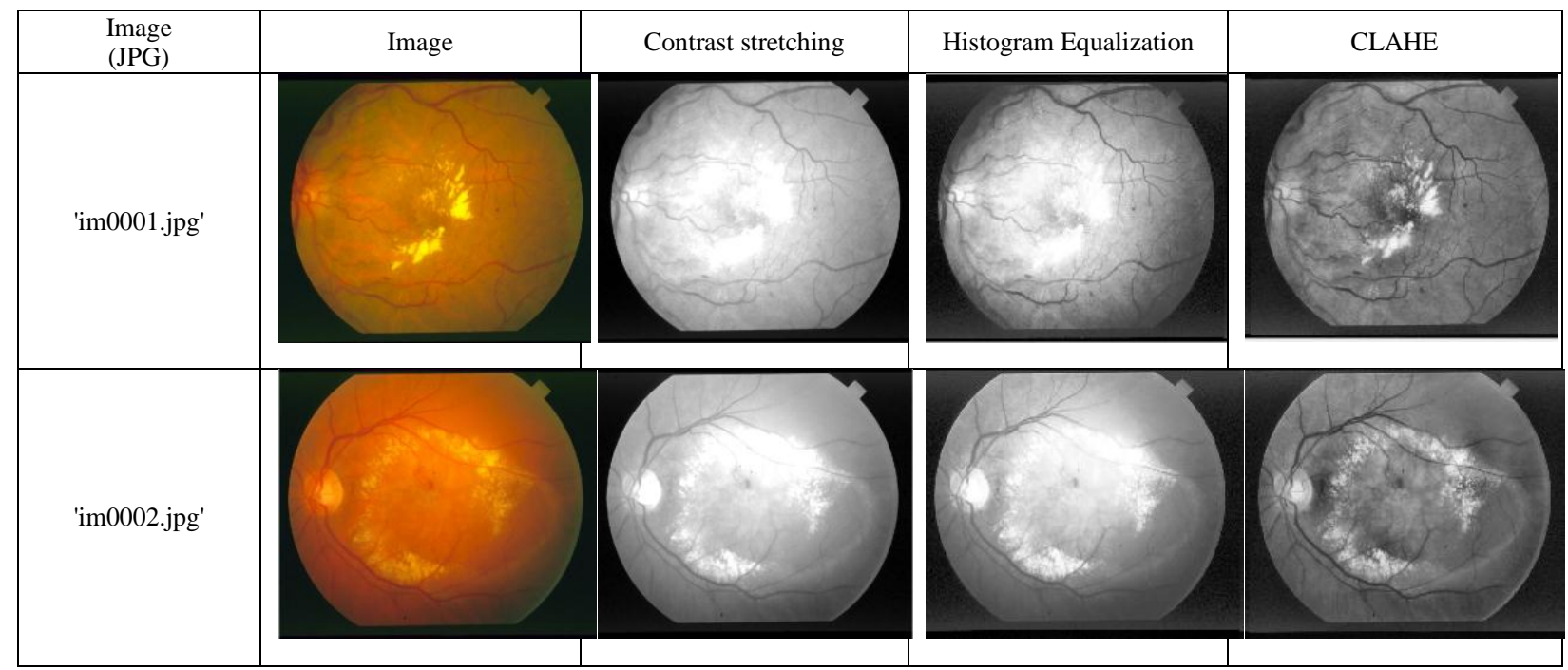




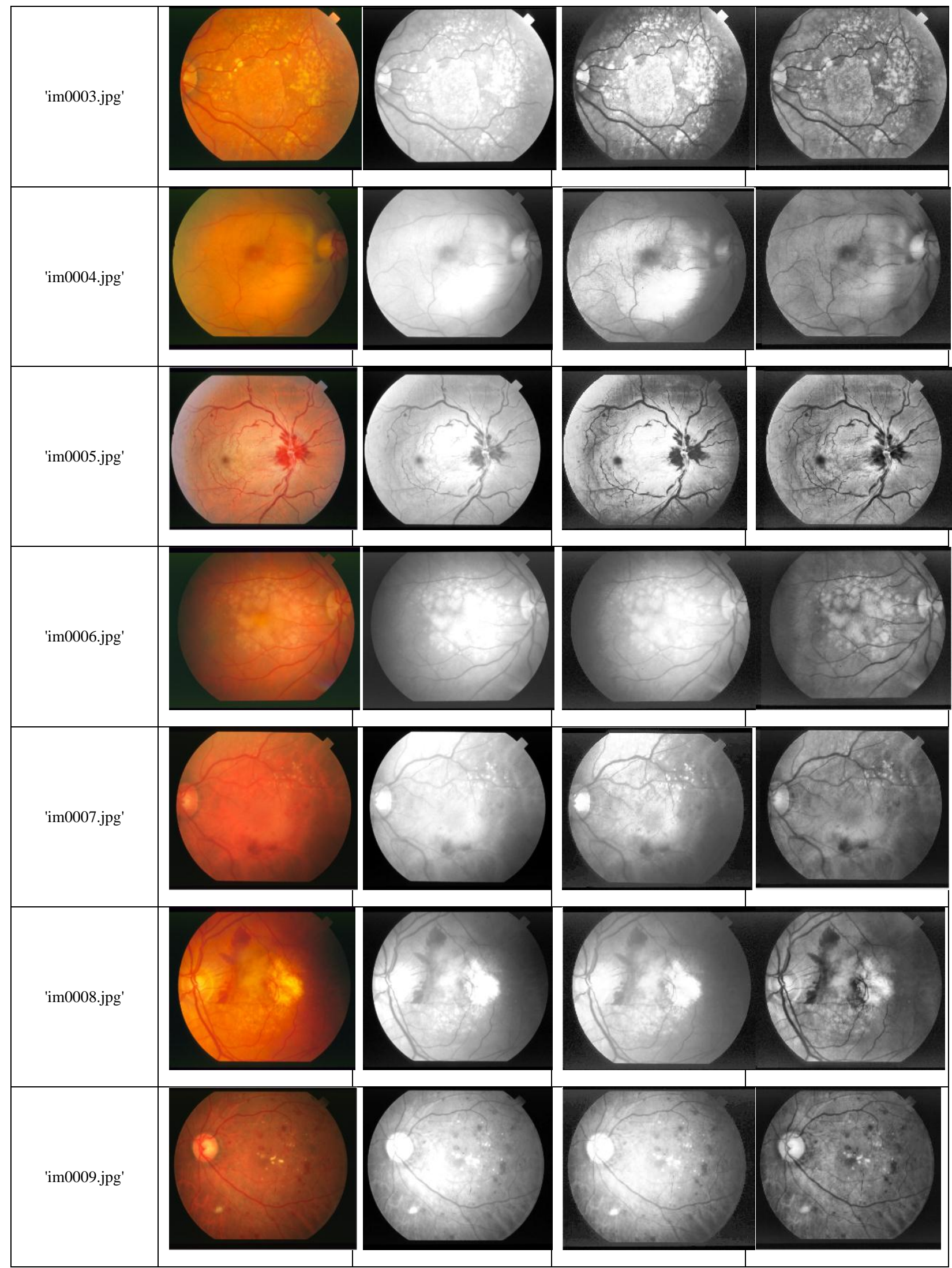




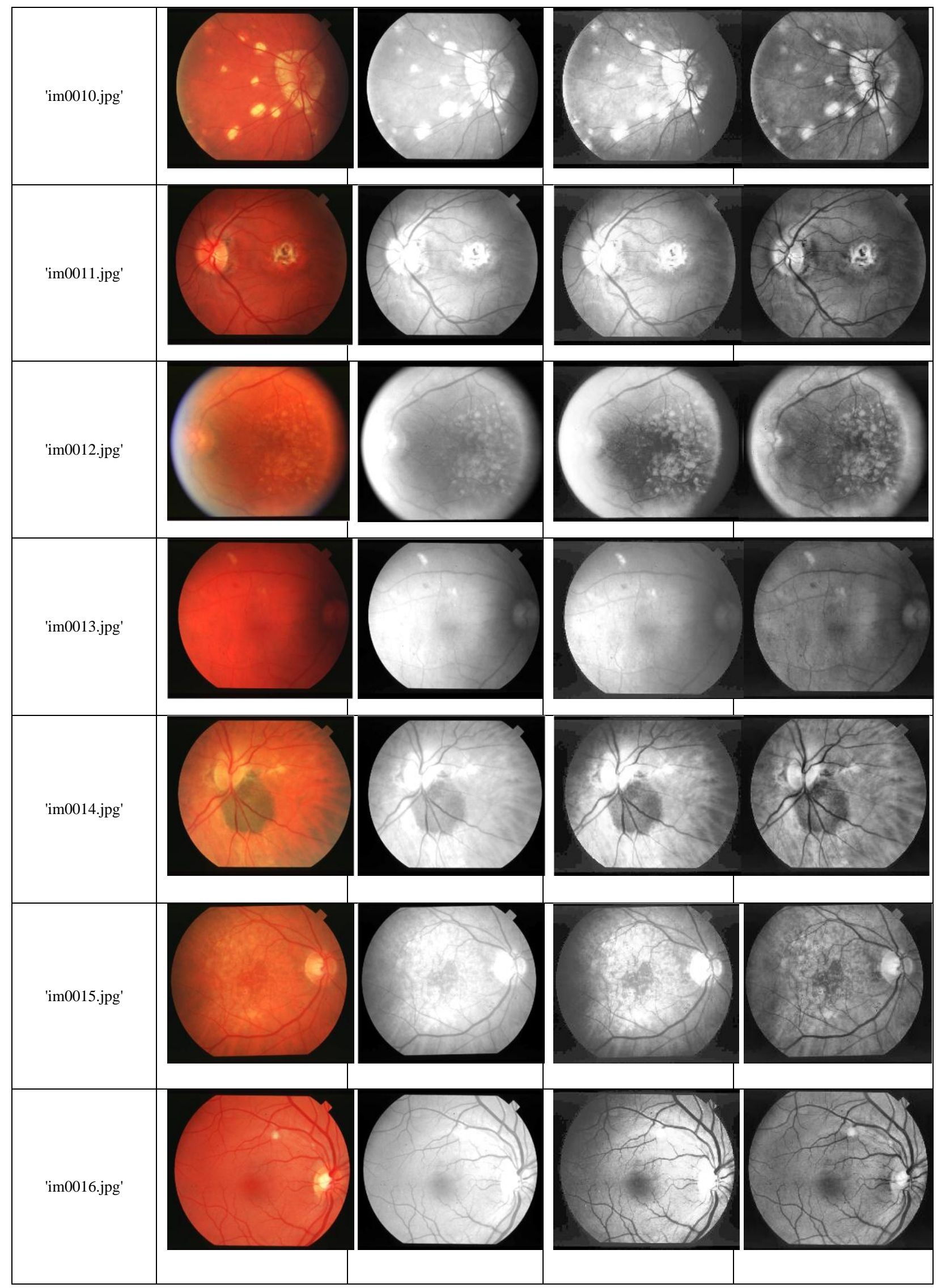




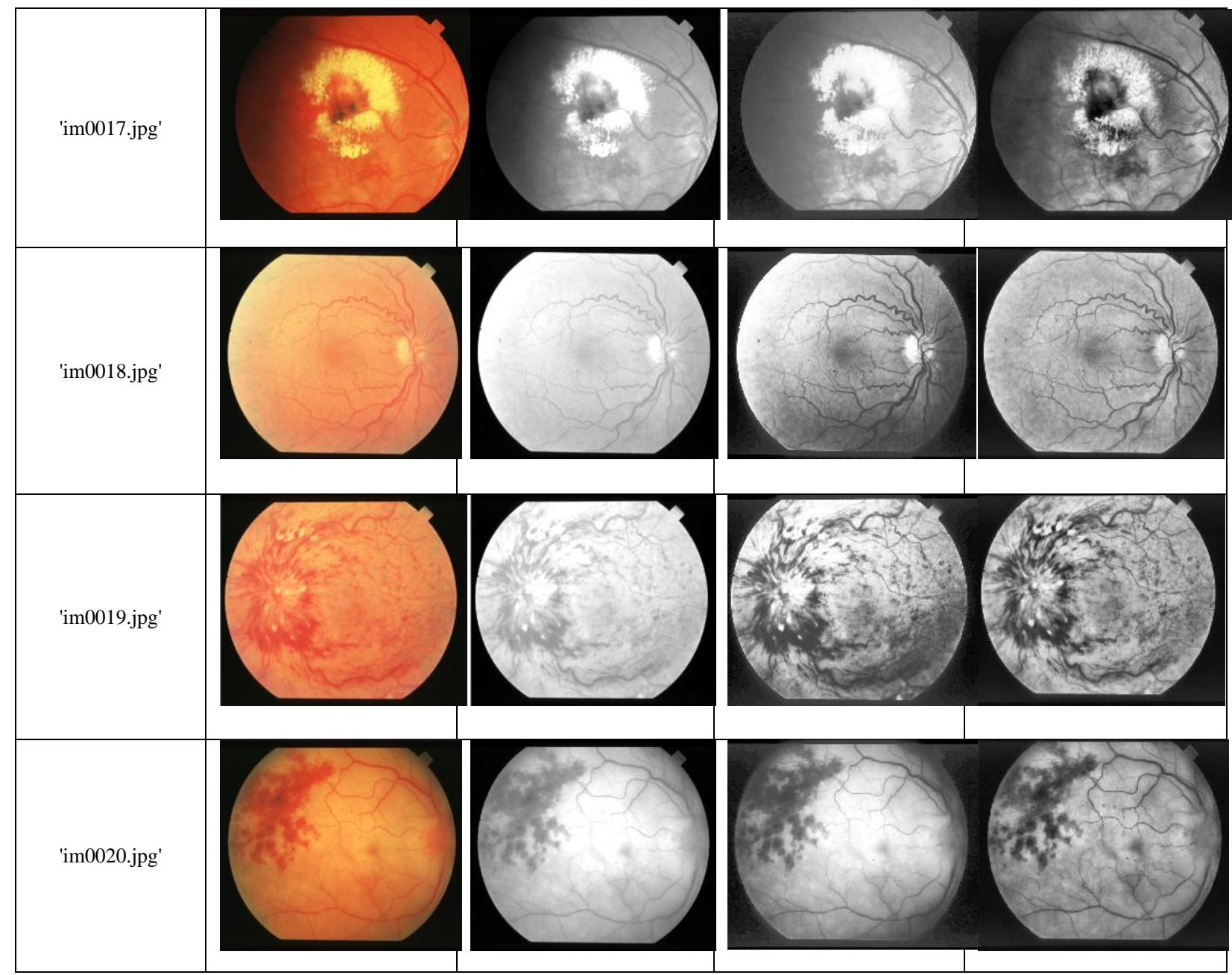

From the results of testing 20 retinal images from the STARE dataset, it can be seen that the initial RGB image was changed to a gray level using a median filter and contrast enhancement. Based on the test results seen from table 1 the results of CLAHE can accentuate blood vessels to be more clear this is in line to improve the quality of the image which will later be continued to the segmentation stage. For the HE results of the test results can be seen in table 1 that blood vessels are visible even if they are not as good as the CLAHE results. And for the results of contrast stretching based on table 1 in terms of highlighting or clarifying the retinal blood vessels are still quite visible. For test results based on table 1 seen from the results of the CLAHE image is a good method in terms of clarifying the blood vessels of the retina.

Table 2. The MSE value of the method is tested with different contrast

\begin{tabular}{|c|c|c|c|}
\hline Image(jpg) & $\begin{array}{c}\text { CS + Median } \\
\text { Filter }\end{array}$ & HE +Median Filter & CLAHE + Filter \\
\hline 'im0001.jpg' & 0.37 & 2.58 & 22.57 \\
\hline 'im0002.jpg' & 0.31 & 0.54 & 23.04 \\
\hline 'im0003.jpg' & 0.50 & 45.24 & 43.91 \\
\hline 'im0004.jpg' & 0.29 & 0.53 & 6.56 \\
\hline 'im0005.jpg' & 3.66 & 53.07 & 41.71 \\
\hline 'im0006.jpg' & 0.33 & 5.19 & 10.50 \\
\hline 'im0007.jpg' & 7.10 & 2.26 & 2.92 \\
\hline 'im0008.jpg' & 0.37 & 0.39 & 5.00 \\
\hline 'im0009.jpg' & 8.47 & 1.23 & \\
\hline
\end{tabular}




\begin{tabular}{|c|c|c|c|}
\hline 'im0010.jpg' & 15.98 & 2.31 & 28.00 \\
\hline 'im0011.jpg' & 10.99 & 1.54 & 6.64 \\
\hline 'im0012.jpg' & 6.77 & 6.89 & 27.29 \\
\hline 'im0013.jpg' & 6.01 & 1.40 & 0.84 \\
\hline 'im0014.jpg' & 14.26 & 11.89 & 34.17 \\
\hline 'im0015.jpg' & 9.44 & 2.67 & 20.25 \\
\hline 'im0016.jpg' & 14.07 & 8.67 & 20.90 \\
\hline 'im0017.jpg' & 21.99 & 2.49 & 48.15 \\
\hline 'im0018.jpg' & 24.57 & 119.95 & 77.28 \\
\hline 'im0019.jpg' & 21.50 & 86.84 & 35.74 \\
\hline 'im0020.jpg' & 15.97 & 18.05 & $\mathbf{2 8 . 4 2}$ \\
\hline Average & $\mathbf{9 . 1 5}$ & $\mathbf{1 8 . 6 9}$ & \\
\hline
\end{tabular}

Table 2. is an MSE of 20 STARE images that use the median filter than with different contrasts, namely, contrast stretching, histogram equalization and CLAHE. From table 2 it can be seen that the results of MSE with contrast stretching and median filter that is with an average of 9.15 are classified as good because the value is below 30. For HE and median filter that is obtained an average of 18.69 with the results of CLAHE and median filter an average of 28.42. From the method tested in this paper, the best MSE value is 9.15 .
In table 3 is the PSNR value of 20 STARE images that use different contrast. It can be seen in table 3 . that the PSNR value is contrast stretching with an average median filter of 42.13. HE and median filter with an average value of 41.33 and for CLAHE with a median filter having an average value of 35.30. From these results, it can be said that the best PSNR value on testing the method with different contrast is 42.14 which is the result of the contrast stretching method with a median filter.

Table 3. The PSNR value of the method was tested with different contrast

\begin{tabular}{|c|c|c|c|}
\hline Image (jpg) & CS + Median Filter & HE +Median Filter & CLAHE + Filter \\
\hline 'im0001.jpg' & 52.47 & 44.05 & 34.63 \\
\hline 'im0002.jpg' & 53.27 & 50.84 & 34.54 \\
\hline 'im0003.jpg' & 51.16 & 31.62 & 31.74 \\
\hline 'im0004.jpg' & 53.48 & 50.94 & 39.99 \\
\hline 'im0005.jpg' & 42.53 & 30.92 & 31.96 \\
\hline 'im0006.jpg' & 52.91 & 41.02 & 37.95 \\
\hline 'im0007.jpg' & 39.65 & 44.62 & 38.20 \\
\hline 'im0008.jpg' & 52.53 & 52.18 & 34.25 \\
\hline 'im0009.jpg' & 38.89 & 47.27 & 41.17 \\
\hline 'im0010.jpg' & 36.13 & 44.53 & 33.69 \\
\hline 'im0011.jpg' & 37.76 & 46.28 & 39.94 \\
\hline 'im0012.jpg' & 39.86 & 39.78 & 33.80 \\
\hline 'im0013.jpg' & 40.38 & 46.70 & 48.91 \\
\hline 'im0014.jpg' & 36.62 & 37.41 & 32.83 \\
\hline 'im0015.jpg' & 38.42 & 43.90 & 35.10 \\
\hline 'im0016.jpg' & 36.68 & 38.78 & 34.96 \\
\hline 'im0017.jpg' & 34.74 & 44.20 & 31.34 \\
\hline 'im0018.jpg' & 34.26 & 27.37 & 29.06 \\
\hline 'im0019.jpg' & 34.84 & 28.78 & 29.28 \\
\hline 'im0020.jpg' & 36.13 & 35.60 & 32.63 \\
\hline Average & 42.14 & 41.40 & 35.30 \\
\hline
\end{tabular}

Based on these three parameters, it can be seen in table 2 and table 3 that the values with different original image contrasts can affect the results of image improvement.
Thus the method proposed in this paper can improve image quality based on the results of MSE and PSNR. 
Table 4. The SSIM value of the method was tested with different contrast

\begin{tabular}{|c|c|c|c|}
\hline Image (jpg) & CS + Median Filter & HE +Median Filter & CLAHE + Filter \\
\hline 'im0001.jpg' & 0.93 & 0.81 & 0.86 \\
\hline 'im0002.jpg' & 0.90 & 0.84 & 0.86 \\
\hline 'im0003.jpg' & 0.89 & 0.77 & 0.82 \\
\hline 'im0004.jpg' & 0.88 & 0.84 & 0.88 \\
\hline 'im0005.jpg' & 0.91 & 0.78 & 0.79 \\
\hline 'im0006.jpg' & 0.89 & 0.86 & 0.89 \\
\hline 'im0007.jpg' & 0.85 & 0.79 & 0.89 \\
\hline 'im0008.jpg' & 0.93 & 0.82 & 0.83 \\
\hline 'im0009.jpg' & 0.83 & 0.71 & 0.86 \\
\hline 'im0010.jpg' & 0.88 & 0.74 & 0.88 \\
\hline 'im0011.jpg' & 0.84 & 0.67 & 0.87 \\
\hline 'im0012.jpg' & 0.93 & 0.83 & 0.87 \\
\hline 'im0013.jpg' & 0.78 & 0.72 & 0.90 \\
\hline 'im0014.jpg' & 0.87 & 0.78 & 0.86 \\
\hline 'im0015.jpg' & 0.87 & 0.77 & 0.86 \\
\hline 'im0016.jpg' & 0.86 & 0.71 & 0.85 \\
\hline 'im0017.jpg' & 0.88 & 0.76 & 0.86 \\
\hline 'im0018.jpg' & 0.87 & 0.77 & 0.85 \\
\hline 'im0019.jpg' & 0.86 & 0.71 & 0.78 \\
\hline 'im0020.jpg' & 0.88 & 0.80 & 0.87 \\
\hline Average & $\mathbf{0 . 8 8}$ & $\mathbf{0 . 7 7}$ & $\mathbf{0 . 8 6}$ \\
\hline & & & \\
\hline & & & \\
\hline
\end{tabular}

In table 4 the results are the SSIM values of the median filter with three different contrasts. Of the three contrasts, the best SSIM values are the results of HE and median filters with an average value of 0.77 . And the average value of CS and median filter is 0.89 while the SSIM CLAHE value and median filter average value is 0.86 . Based on the three parameters that can be seen in table 2, table 3 , and table 4 that the value with different original image contrast can affect the results of image improvement. Thus the method proposed in this paper can improve image quality based on the results of MSE and PSNR, the best value in SSIM is in the HE method and median filter.

Table 5. Comparison of MSE and PSNR values in the method tested with the previous method to improve image quality

\begin{tabular}{|c|c|c|c|}
\hline Method & MSE & PNSR & SSIM \\
\hline $\begin{array}{c}\text { Proposed Method } \\
\text { (CS + Median } \\
\text { Filter) }\end{array}$ & $\mathbf{9 . 1 4}$ & $\mathbf{4 2 . 1 3}$ & $\mathbf{0 . 8 7}$ \\
\hline $\begin{array}{c}\text { Proposed Method } \\
\text { (HE + Median } \\
\text { Filter) }\end{array}$ & $\mathbf{1 8 . 6 8}$ & $\mathbf{4 1 . 3 9}$ & $\mathbf{0 . 7 7}$ \\
\hline $\begin{array}{c}\text { Proposed Method } \\
\text { (CLAHE + Median } \\
\text { Filter) }\end{array}$ & $\mathbf{2 8 . 4 2}$ & $\mathbf{3 5 . 2 9}$ & $\mathbf{0 . 8 5}$ \\
\hline $\begin{array}{c}\text { (S. Sahu et al, } \\
\text { 2018) [22] }\end{array}$ & - & 35.37 & 0.96 \\
\hline $\begin{array}{c}\text { (Zulfahmi et al, } \\
\text { 2019) [23] }\end{array}$ & 85.52 & 28.88 & - \\
\hline $\begin{array}{c}\text { (B. Gupta et al, } \\
\text { 2018) [21] } \\
\text { HE }\end{array}$ & - & 25.79 & 0.56 \\
\hline $\begin{array}{c}\text { (B. Gupta et al, } \\
\text { 2018) [21] } \\
\text { CLAHE }\end{array}$ & - & 9.48 & 0.33 \\
\hline
\end{tabular}

Copyright (C) 2020 MECS
Based on table 5 it can be seen that the comparison of the results of the method being tested is better contrasted stretching with the median filter because the values of MSE and PSNR are better. Because the closer to zero the MSE results of an image processing result, the better. Whereas the PSNR value is more than $35 \mathrm{~dB}$, the better the results of image quality improvement. However, the SSIM value proposed by S.Sahu, et al [22] is superior. Because the SSIM value will get better if it gets closer to zero. From the results of these comparisons, the method being tested can match the results of the process from previous results.

\section{CONCLUSION}

Based on the method proposed in this paper using the STARE dataset by taking retinal images in JPG format. Of the images, image quality improvements were made using three contrasts, contrast stretching, HE and CLAHE filtered using a median filter. With the first comparison using the proposed method contrast stretching and median filter. The technique of taking maximum and minimum values using stretchlim which was previously the image in grayish conditions. HE and median filters with equalization techniques on histograms whose pixel values are at 0 to 255 . The third is CLAHE and median filters which technically limit the pixel values to the boundary regions. The method proposed in this paper can improve image quality based on MSE and PSNR results. From the results of testing with three different methods and based on three different parameters, the best MSE and PSNR results are contrasted stretching with values of 9.14 and $42.13 \mathrm{~dB}$. Thus contrast stretching is better in 
improving image quality. In the future, it is expected to improve the image quality with the contrast stretching method with fixed results as an RGB image. With the CLAHE method in the section to clarify the blood vessels of the retina image very well, it would be better if it continued with image segmentation in the future.

\section{REFERENCES}

[1] M. Rajaram, "A novel approach for contrast enhancement based on histogram equalization followed by median filter," ARPN J. Eng. Appl. Sci., no. September 2009, 2014.

[2] V. M. Saffarzadeh, A. Osareh, and B. Shadgar, "Vessel Segmentation in Retinal Images Using Multi - scale Line Operator and K - Means Clustering," Dep. Comput. Eng. Shahid Chamran Univ. Ahvaz, Khuzestan, Iran,jmss., vol. 4, no. 2, 2017.

[3] H. Aguirre-ramos, J. G. Avina-cervantes, I. Cruz-aceves, J. Ruiz-pinales, and S. Ledesma, "Blood vessel segmentation in retinal fundus images using Gabor filters , fractional derivatives , and Expectation Maximization," Appl. Math. Comput., vol. 339, pp. 568-587, 2018.

[4] F. Farokhian and H. Demirel, "Blood Vessels Detection and Segmentation in Retina using Gabor Filters," Electr. Electron. Eng. Dep. IEEE, pp. 104-108, 2013.

[5] K. Firdausy, T. Sutikno, and E. Prasetyo, "Image Enhancement Using Contrast Stretching On Rgb And Ihs Digital Image," Cent. Electr. Eng. Res. Solut. (CEERS), ISSN, vol. 5, No.1, no. 1, pp. 45-50, 2007.

[6] Y. Zhu and C. Huang, "An Improved Median Filtering Algorithm for Image Noise," Phys. Procedia, vol. 25, pp. 609-616, 2012.

[7] F. A. Jassim and F. H. Altaani, "Hybridization of Otsu Method and Median Filter for Color Image Segmentation," Int. J. Soft Comput. Eng. ISSN 2231-2307, vol. Volume-3, no. 2, pp. 69-74, 2013.

[8] P. Garhwal and P. Garhwal, "A Hybrid Approach to Image Enhancement using Contrast Stretching on Image Sharpening and the analysis of various cases arising using Histogram," IEEE Int. Conf. Recent Adv. Innov. Eng., no. 3, 2014.

[9] B. Xu, Y. Zhuang, H. Tang, and L. Zhang, “Object-Based Multilevel Contrast Stretching Method for Image Enhancement," IEEE Trans. Consum. Electron., vol. 56, no. 3, pp. 1746-1754, 2010.

[10] R. K. B, H. Kabir, and S. Salekin, "Contrast Enhancement by Top-Hat and Bottom-Hat Transform with Optimal Structuring Element: Application to Retinal," Dep. Comput. Sci. Eng., pp. 533-540, 2017.

[11] M. Liao, Y. Zhao, X. Wang, and P. Dai, "Retinal vessel enhancement based on multi-scale top-hat transformation and histogram fitting stretching," Opt. Laser Technol., vol. 58, pp. 56-62, 2014.

[12] P. P. Acharjya and S. Mukherjee, "Digital Image Segmentation Using Median Filtering and Morphological Approach," Int. J. Adv. Res. Comput. Sci. Softw. Eng., vol. 4, no. 1, pp. 552-557, 2014.

[13] K. B. Khan, A. A. Khaliq, A. Jalil, and M. Shahid, "A robust technique based on VLM and Frangi filter for retinal vessel extraction and denoising," PLoS One, vol. 13, no. 2, pp. 1-22, 2018.

[14] A. M. Reza, "Realization of the contrast limited adaptive histogram equalization (CLAHE) for real-time image enhancement," J. VLSI Signal Process. Syst. Signal Image. Video Technol., vol. 38, no. 1, pp. 35-44, 2004.
[15] H. A. Rahim, A. S. Ibrahim, W. M. D. W. Zaki, and A. Hussain, "Methods to Enhance Digital Fundus Image for Diabetic Retinopathy Detection," IEEE Int. Colloq. Signal Process. its Appl., no. Md, pp. 7-9, 2014.

[16] S. Anitha and V. Radha, "Contrast Stretching and Non Linear Median Filters for Fabric Inspection," Int. J. Comput. Sci. Inf. Technol., vol. 2, no. 2, pp. 836-839, 2011.

[17] S. S. Al-amri, N. V Kalyankar, and S. D. Khamitkar, "Linear and Non-linear Contrast Enhancement Image," IJCSNS Int. J. Comput. Sci. Netw. Secur. VOL.10, vol. 10, no. 2, pp. 139-143, 2010.

[18] G. B. Iwasokun and O. C. Akinyokun, "Enhancement Methods : A Review," Sci. Int., vol. 4, pp. 2251-2277, 2016.

[19] J. Dash, "Retinal Blood Vessel Segmentation Using Otsu Thresholding With Principal Component Analysis," 2018 2nd Int. Conf. Inven. Syst. Control, no. Icisc, pp. 933-937, 2018.

[20] S. Shrestha, "I MAGE D ENOISING U SING N EW A DAPTIVE," Signal Image Process. An Int. J., vol. 5, no. 4, pp. 1-13, 2014.

[21] B. Gupta and M. Tiwari, "Color retinal image enhancement using luminosity and quantile based contrast enhancement," PDPM Indian Inst. Inf. Technol., 2018.

[22] S. Sahu, A. Kumar, S. P. Ghrera, and M. Elhoseny, "An approach for de-noising and contrast enhancement of retinal fundus image using CLAHE," Opt. Laser Technol., 2018.

[23] Erwin, R. Zulfahmi, G. Utami, A. Harison, D. Noviyanti, and P. Agung, "Improved Image Quality Retinal Fundus with Contrast Limited Adaptive Histogram Equalization and Filter Variation," 2019 International Conference on Informatics, Multimedia, Cyber and Information System (ICIMCIS), vol. 2019, Jakarta, Indonesia, pp. 156-161, 2019.

\section{Authors' Profiles}

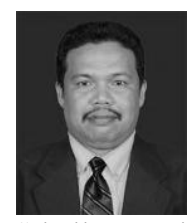

Name :

Erwin

\section{Affiliation:}

Associate Professor at Department of Computer Systems, Faculty of Computer Science, Sriwijaya University, Indonesia.

\section{Address:}

J1. Srijaya Negara, Bukit Lama, Kec. Ilir Bar. I, Kota Palembang, Sumatera Selatan 30128, Indonesia

\section{Brief Biographical History:}

1994 Received the Bachelor degree in Mathematics from the University of Sriwijaya, Indonesia

2002 Received the M.Sc. degrees in Actuarial from the Bandung Institute of Technology (ITB), Bandung, Indonesia 2019 Received the Doctorate in Engineering, Faculty of Engineering, Sriwijaya University, Indonesia

\section{Main Works:}

Current research interests are image processing and computer vision.

Membership in Academic Societies:

- International Journal of Applied Mathematics (IAENG) 
- Institute of Electrical and Electronics Engineers (IEEE)

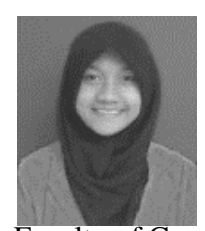

Name :

Dwi Ratna Ningsih

\section{Affiliation:}

Student at Department of Computer Systems, Faculty of Computer Science, Sriwijaya University, Indonesia

\section{Address:}

Jl. Tamyiz Timbangan, Indralaya, Ogan Ilir, Kota Palembang, Sumatera Selatan 30264, Indonesia

\section{Brief Biographical History:}

2016- Pursuing bachelor's degree at Department of Computer Systems, Faculty of Computer Science, Sriwijaya University, Indonesia

How to cite this paper: Erwin, Dwi Ratna Ningsih, " Improving Retinal Image Quality Using the Contrast Stretching, Histogram Equalization, and CLAHE Methods with Median Filters", International Journal of Image, Graphics and Signal Processing(IJIGSP), Vol.12, No.2, pp. 30-41, 2020.DOI: 10.5815/ijigsp.2020.02.04 